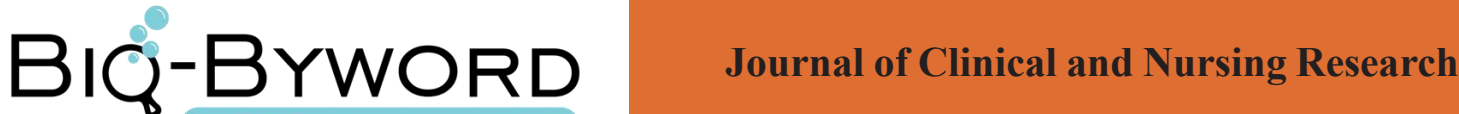

\section{Analysis of Causes and Treatment of Biliary Leakage after Hepatobiliary Surgery}

\author{
Hua Liu, Xiaowei Yang, Wenjie Yan
}

Wuxi Huishan District People's Hospital, Wuxi, Jiangsu Province, 214187, China

\section{ARTICLE INFO}

Article history:

Published online: $30^{\text {th }}$ Sept, 2017

\section{Key words:}

hepatobiliary surgery

causes of biliary leakage

treatment

\begin{abstract}
Hepatobiliary surgery is very common, and its operation is mainly based on the principle of human anatomy to carry out operations. In the actual operation, if there are no corresponding protective measures, it is easy to damage bile tract and bile duct, even biliary leakage complications will be caused after the surgery, and a certain effect will have on the patient's body and mind. It is necessary to strengthen the prevention and treatment of biliary leakage on the basis of thinking on the causes of bile leakage, and it can reduce the chance of the complications. Therefore, this article mainly focuses on strengthening the analysis and discussion on the cause of biliary leakage after hepatobiliary surgery and the treatment of related aspects.
\end{abstract}

Hepatobiliary surgery is a common surgical operation, and after the hepatobiliary surgery, patients prone to have various types of complications, among which biliary leakage is more common. For patients with biliary leakage, if the effective diagnosis measures and treatment measures are not taken in time, the life and health of the patients will be jeopardized. Therefore, we must find out the cause of the patients' biliary leakage as early as possible and take effective treatment plan to avoid the harm to the patients' health caused by the treatment of biliary leakage.

\section{Analysis of causes of biliary leakage after hepatobiliary surgery}

Biliary leakage mainly refers to the patient's bile or its liquid has not been discharged by the normal way. The relevant research shows that biliary leakage is a very common complication after hepatobiliary surgery, and will also affect the health of patients in serious situation. The main causes of biliary leakage after hepatobiliary surgery are: the patients have a certain omission in the value of the accessory liver tube after the operation, the T-tube position produces errors, the patients' physical quality is not good, and other diseases. If the biliary leakage problem after hepatobiliary surgery is not dealt with in time, it is often very easy to produce infection problems, so that most organs in the body appear to be depleted and abnormal, and it even will often cause threat to the patient's life in serious situation. The problem of omission or injury caused by the patients' accessory hepatic duct is also caused by the injury to the structure of the accessory liver duct of bile duct, resulting in the local adhesion and the corresponding inflammation. Ambiguity in the initial stage of the anatomy of the structure of secondary hepatic duct can effect the surgery on the observation of the accessory hepatic duct and make the doctor fail in the surgical operation of the cystic duct for some of the lower openings and also fail in finding and dealing with the problem in the actual operation. Secondly, T-Tube position failure: before the placement of T-tube in the operation, ensure that the doctor gives priority to the materials, cut, and diameter of the T-tube; in the T-tube placement, make sure there's no problem in the position and the implementation of the stitching, so that the $\mathrm{T}$ tube after the completion of the placement will not affect the function and the biliary leakage will not be caused. Thirdly, the patient's own physical condition. Because the patient's body condition can cause corresponding biliary leakage in the hepatobiliary surgery. And some patients have biliary leakage because other related disease decreases their body resistance.

In actual clinical practice, hepatobiliary surgery is very common. In the operation patient's own biliary system usually needs to be resected, drained, sutured, etc., and biliary leakage is easy to be caused after the completion 
of the operation, effecting the the body recovery and quality of life of patients after the operation. According to relevant studies, there are many causes of biliary leakage. For example, the clinical physician is not well aware of the procedure, resulting in improper treatment after the operation and the cause of biliary leakage. If the actual causes of patients' biliary leakage after hepatobiliary surgery is looked for and treated rightly, and be drained in time, then it's possible to prevent pelvic inflammatory disease or bile peritonitis or other production and ensure patients' life safety in the actual treatment and recovery.

Because many biliary leakage problems are caused in the operation of hepatobiliary surgery, therefore, in the clinical, we need to ensure that prevention measures are taken for the cause of biliary leakage during the operation, analyze the causes in accordance with actual experience, and make the relevant measures comprehensive and standardized, reducing the cause of biliary leakage fundamentally. Therefore, the clinician's professional level needs to be strengthened continuously, the actual operation ability needs to be strengthened and understand, the corresponding preparation work needs to be done before the operation to guarantee the implementation in the operation in accordance with the corresponding specification, and the corresponding prevention work needs to be done after the surgery, preventing the cause of biliary leakage from its root.

\section{Analysis of specific measures for treatment}

It is common to use ERCP to diagnose the biliary leakage problem after the operation, and it is a preferred way of doing so after the problem arises. In the actual clinical treatment, the same way should be used to strengthen treatment in accordance with the actual situation of patients. In the second treatment of the patient, we have to know the relevant indications in the actual treatment to reduce the patient's pain, so that the disease can quickly get effective control, thereby improving the quality of life of patients. For patients without drainage tube after the completion of the operation, it is in the production of fever and abdominal pain and other symptoms, it is best to implement the drainage tube setting at the residual end of the bile duct, and implement the restoration after the condition is stable. Blind implementation is prohibited in the operation.

After the hepatobiliary surgery, the drainage tube's indwelling must be carried out in accordance with the actual requirements of the patient. At the same time, must strengthen the rational remedy and treatment for the related complications of patients after the operation. And on this basis, the ERCP examination also needs to be strengthened, so as to reduce the probability of biliary leakage after the operation and increase the cure rate.

In short, in the clinical operation, biliary leakage phenomenon can be caused because because the doctor's surgical technique is not very high or the mistakes in the operation, and we need to carry out the targeted treatment for the problem, so that the probability of biliary leakage after the operation can be reduced. Therefore, it needs to be applied effectively and promoted in the clinical.

\section{References}

[1]Miguel A. Cuesta. Case on Bile Leakage (and Portal Vein Thrombosis) After Whipple Procedure[M].Springer International Publishing:2014.

[2]Yo-ichi Yamashita, Takayuki Hamatsu, Tatsuya Rikimaru, Shinji Tanaka, Ken Shirabe, Mitsuo Shimada, Keizo Sugimachi. Bile Leakage After Hepatic Resection[J]. Annals of Surgery, 2001, 233(1):.

[3]Yasuhiko Nagano, Shinji Togo, Kuniya Tanaka, Hidenori Masui, Itaru Endo, Hitoshi Sekido, Kaoru Nagahori , Hiroshi Shimada. Risk Factors and Management of Bile Leakage after Hepatic Resection[J]. World Journal of Surgery, 2003， 27(6):

[4]L. Morgenstern, G. Berci, E. H. Pasternak. Bile leakage after biliary tract surgery[J]. Surgical Endoscopy, 1993, 7(5):.

[5]K. Hanazaki, J. Igarashi, H. Sodeyama, Y. Matsuda. Bile leakage resulting from clip displacement of the cystic duct stump[J]. Surgical Endoscopy, 1999, 13(2):.

[6]Takahiro Kajiwara, Yutaka Midorikawa, Shintaro Yamazaki, Tokio Higaki, Hisashi Nakayama, Masamichi Moriguchi, Shingo Tsuji, Tadatoshi Takayama. Clinical score to predict the risk of bile leakage after liver resection[J]. BMC Surgery, 2016, 16(1):.

[7]K. Radecke, H. Lang, M. Malagó, G. Gerken, U. Treichel. Successful Sealing of Persisting Postoperative Bile Leakages by Fibrin Glue[J]. Endosk heute, 2004, 17(04):

[8]Hoekstra Lisette T, van Gulik Thomas M, Gouma Dirk J, Busch Oliver R. Posthepatectomy bile leakage: how to manage.[J]. Digestive Surgery, 2012, 29(1):

[9]Nagano Yasuhiko, Togo Shinji, Tanaka Kuniya, Masui Hidenori, Endo Itaru, Sekido Hitoshi, Nagahori Kaoru, Shimada Hiroshi. Risk factors and management of bile leakage after hepatic resection.[J]. World Journal of Surgery, 2003, 27(6):.

[10]Capussotti Lorenzo , Ferrero Alessandro, Viganò Luca, Sgotto Enrico, Muratore Andrea, Polastri Roberto. Bile leakage and liver resection: Where is the risk?[J]. Archives of Surgery, 2006, 141(7):. 